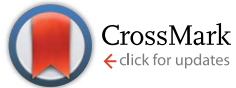

Cite this: RSC Adv., 2017, 7, 9981

Received 16th December 2016 Accepted 20th January 2017

DOI: $10.1039 / c 6 r a 28298 a$

rsc.li/rsc-advances

\section{Fabrication of a superhydrophobic surface with a hierarchical nanoflake-micropit structure and its anti-icing properties}

\begin{abstract}
Guanyu Wang, ${ }^{a}$ Yizhou Shen, ${ }^{\text {ab }}$ Jie Tao, ${ }^{* a}$ Xinyi Luo, ${ }^{a}$ Liqing Zhang ${ }^{c}$ and Yiping Xia ${ }^{a}$
Herein, we report a novel route to fabricate a robust anti-icing superhydrophobic surface with a hierarchical nanoflake-micropit structure (constructed by a combination of lithography processing and chemical etching methods) on an aluminum substrate. Moreover, the superhydrophobicity and icephobicity were systematically explored based on a comparative study with different structured surfaces prepared under four different processing conditions. Results indicated that the icephobicity and superhydrophobicity of the hierarchically structured surfaces were superior to those of the single-structured surfaces, and the superhydrophobic hierarchically structured surface exhibited high non-wettability, with an apparent contact angle as high as approximately $173^{\circ}$ and a sliding angle as low as about $4.5^{\circ}$. In addition, for this surface, ice adhesion strength was greatly reduced to $75 \mathrm{kPa}$. Therefore, we reasonably believe that the superhydrophobic surface with a hierarchical nanoflake-micropit structure possesses an excellent water repellency and excellent anti-icing properties, displaying an ideal platform for applications in the aviation industry.
\end{abstract}

\section{Introduction}

Aircraft anti-icing/de-icing technologies have been an important research field, eliciting wide interest due to significant economic, energy and safety issues in the aircraft industry. ${ }^{1}$ Ice accumulates on aircrafts mainly on the exposed surfaces, such as the horizontal tail, vertical tail and engine vacuum cover, and has already been recognized as a serious problem that threatens flight safety and stability. It has been demonstrated that ice formation and accretion on the surface of an aircraft is mainly caused due to a large amount of supercooled water droplets aggregating and freezing, particularly when the aircraft flies through supercooled clouds. ${ }^{2}$

The well-known traditional passive anti-icing/de-icing methods to melt away ice include treatment with chemicals such as glycol, mechanical vibration to break already-formed ice layers, and electrothermal treatment. ${ }^{3}$ However, these methods cannot effectively prevent and remove ice accumulation or frost formation on an aircraft, particularly in hostile environments, such as freezing rain, snow, in-cloud icing or fog icing. ${ }^{4}$ Furthermore, current industrial strategies to combat icing still

${ }^{a}$ College of Materials Science and Technology, Nanjing University of Aeronautics and Astronautics, Nanjing 210016, P. R. China. E-mail: shenyizhou@nuaa.edu.cn; taojie@nuaa.edu.cn; Fax: +865211 2911; Tel: +86 52112911

${ }^{b}$ Aerospace Engineering College, Nanjing University of Aeronautics and Astronautics, Nanjing 210016, P. R. China

${ }^{c}$ School of Materials Science \& Engineering, South-east University, Nanjing 211189, P. R. China present several shortcomings, such as additional energy consumption, increased flight operation cost, and environmental pollution. ${ }^{5,6}$

On another research line, extensive progress has been made in the development of active anti-icing/de-icing methods by incorporating micro- or nano-scale materials with a rough texture and heterogeneous hydrophobic surfaces with low surface energy. Inspired by the lotus leaf, peanut leaf, cicada wing, and butterfly wing, some researchers have tried to visualize and replicate the structure of those special surface topographies, so that they are capable of artificially producing superhydrophobic surfaces. The corresponding micromechanism for these surfaces was also proposed, suggesting that the micro- or nano-scale textures could capture small air pockets, making them interconnected so as to evolve into an integrated air layer. ${ }^{7}$ The superhydrophobic solid surfaces with more ample areas for air entrapment not only sustain a stable insulation or reduce the solid-liquid contact area by uplifting the droplets, but also exert a lubrication force that promotes bouncing off from the flat hydrophobic solid surface before ice nucleation can occur. ${ }^{8}$ Moreover, the air layer can form a thermal barrier between the surface and the supercooled water droplets to greatly reduce thermal conversion efficiency. This effectively prevents heat transfer as well as decreases icing nucleation, and thus delays icing time. ${ }^{\mathbf{9 , 1 0}}$

There are previous reports describing the preparation of superhydrophobic surfaces. Wenjie Zhao et al. ${ }^{\mathbf{1 1}}$ manipulated the etching pits and convex structure arranged regularly via chemical etching, anodic oxidation and self-assembly of PTES 
on $\mathrm{Al}$ alloys. The results showed that continuous and highly ordered nanopores formed a porous layer of anodic oxide film, which was able to enhance the air trapping capability of the surface. This caused the surface to exhibit superhydrophobicity, with an apparent water contact angle of $156^{\circ}$ and lower adhesion than that of other samples after chemical modification. Qinghua Zhang et al. ${ }^{12}$ fabricated a fluorinated magnetite nanocomposite with covalent integration, possessing a rough micro/nano-structure and stable superhydrophobic properties. At low temperatures $\left(-15{ }^{\circ} \mathrm{C}, \mathrm{RH}: 50 \pm 5 \%\right)$, the superhydrophobic surface could delay the freezing time from $50 \mathrm{~s}$ to $2878 \mathrm{~s}$. The ice adhesion strength was greatly reduced compared with that of a pure copolymer coating. The latest report from Boinovich's group ${ }^{\mathbf{1 3}}$ claimed that superhydrophobic coatings fabricated by intense nanosecond-pulsed laser irradiation and chemical surface hydrophobization of an aluminum alloy displayed excellent icing-delay properties, and the freezing delay time reached more than 6 hours at $-20{ }^{\circ} \mathrm{C}$. These researchers have made some progress in the development of various superhydrophobic surfaces with improved anti-icing properties. However, most studies are focused on the construction of antiicing superhydrophobic surfaces in a research setting, neglecting the industrial requirements for practical applications, such as relatively low cost, time-efficiency, and simple fabrication processes.

In this study, we fabricated a robust superhydrophobic surface with a hierarchical nanoflake-micropit structure (constructed by the combination of lithography processing and chemical etching method) on an aluminum substrate via modification with fluoroalkylsilane (FAS-17). The present study focused on the systematic characterizations of surface morphology and composition, and the study of the static/ dynamic contact angles of an impacting water droplet. In addition, the icephobic potential of the superhydrophobic surfaces was studied via static analysis of the ice adhesion strength and the analysis of the dynamic contact process of an impacting supercooled water droplet at lower temperatures.

\section{Experimental}

\section{Materials}

Aluminium substrates (99.9\%) were obtained from the Beijing Nonferrous Metal Research Institute, China. These were cut into small pieces with the size of $15 \mathrm{~mm} \times 15 \mathrm{~mm} \times 2 \mathrm{~mm}$. SU-8 photoresist was purchased from Gersteltec Sarl Co., Ltd, Switzerland. All the chemicals used in this experiment, such as $\mathrm{NaOH}, \mathrm{NaNO}_{3}$, acetone and ethanol, were of analytical grade and were provided by Sinopharm Chemical Reagent Co., Ltd, China. Ultrapure deionized water was obtained from an Ulupure-II-20T water system (Chengdu, China) and used throughout the experiments. In addition, as a modifying agent, commercial grade heptadecafluorodecyl trimethoxysilane (FAS17, purchased from Tokyo chemical industry Co., Ltd, Japan) was used to modify the micro/nano-hierarchically structured surfaces.

\section{Sample preparation}

Herein, we introduced a very economic and simple method to construct a superhydrophobic surface on an Al substrate. The entire process to fabricate a superhydrophobic hierarchically structured surface is divided into four steps:

(1) Lithography process to obtain a regular microscale pattern on an Al substrate in a controlled manner;

(2) Alkali etching treatment to induce the formation of an aluminum hydroxide $\left(\mathrm{Al}(\mathrm{OH})_{3}\right)$ layer;

(3) Water boiling treatment to construct particular nanostructures;

(4) Coating with a self-assembled monolayer of low surface energy.

First, the Al substrates were successively ultrasonically cleaned in acetone, alcohol, and deionized water for $15 \mathrm{~min}$ and then dried under cold air. Subsequently, these samples were mechanically polished to obtain a flat surface that met the basic requirement for a lithography process. The mask template used in the lithography process was divided in two sections: a dark square section for the exposed area, and a light-coloured section for the non-exposed area, as shown in Fig. 1(a). Then, a photoresist film with a thickness of $5 \mu \mathrm{m}$ was selectively exposed using a standard UV mask aligner, and developed to have the initial pattern on the pretreated Al substrate covered by the SU-8 photoresist. Finally, the substrate was immersed in $100 \mathrm{ml}$ of $10 \% \mathrm{NaNO}_{3}$ aqueous solution in an electrochemical cell for $2 \mathrm{~min}$ at a voltage of $10 \mathrm{~V}$. After stripping and rinsing, the controllable regular microscale patterns could be obtained as shown in Fig. 1(b).

\section{(a)}
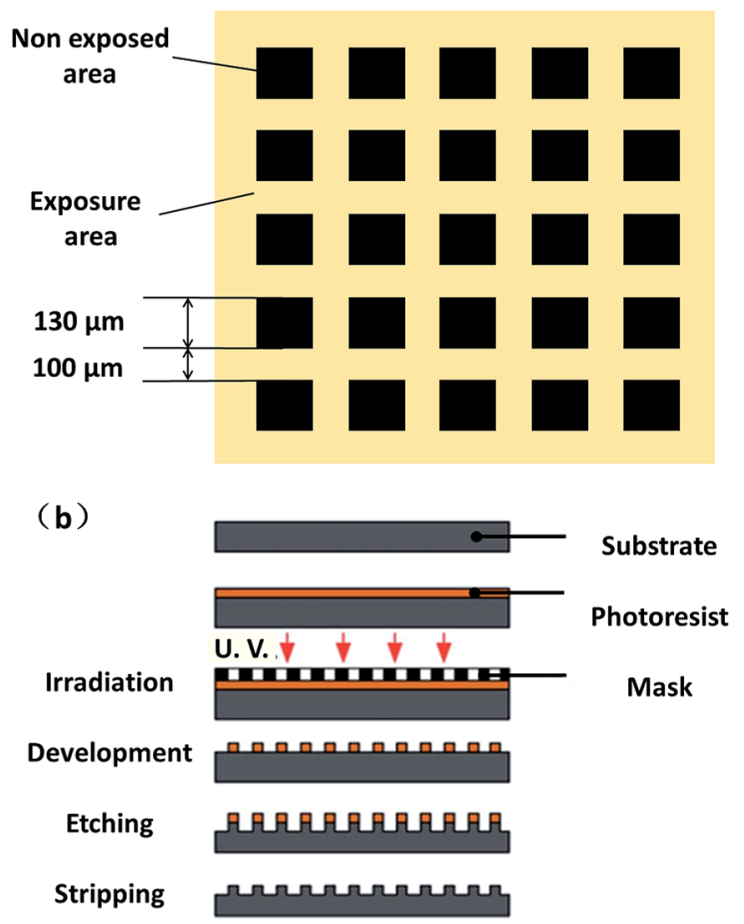

Fig. 1 (a) Design of the mask template used in the lithography process; (b) micromachining process for the controllable regular microscale pattern. 
To achieve a micro/nano-structure, i.e., a layer of nanoflakes deposited on the surface of the regular array pattern, the samples were placed in $100 \mathrm{ml}$ of $0.05 \mathrm{M} \mathrm{NaOH}$ aqueous solution inside an oil-bath furnace at $80{ }^{\circ} \mathrm{C}$ for $5 \mathrm{~min}$ in the open air. Then, the samples were immediately rinsed with deionized water and immersed in $100 \mathrm{ml}$ of boiling deionized water for $40 \mathrm{~min}$ in an oil-bath furnace. After the two-step immersion process, the samples were modified in a $1 \mathrm{wt} \% \mathrm{FAS}-17$ ethanolic solution for $24 \mathrm{~h}$ and then dried in an oven at $120{ }^{\circ} \mathrm{C}$ for $2 \mathrm{~h}$ to obtain the superhydrophobic hierarchically structured surface.

Furthermore, we designed four types of surfaces for a comparative study of their anti-icing properties: Al substrate surface with FAS-17 modification (sample 1), micropitstructured surface with FAS-17 modification (sample 2), nanoflake-structured surface with FAS-17 modification (sample 3 ), and hierarchically structured (micropit regular array and nanoflakes) surface with FAS-17 modification (sample 4).

\section{Surface topography and chemical composition}

Sample morphologies were characterized via field emission scanning electron microscopy (FE-SEM; Hitachi S4800, Japan). An atomic force microscope (AFM; Dimension Edge from Bruker, Germany) was used for examining surface topography. AFM was conducted in tapping mode with a scanning range of 2 $\mu \mathrm{m} \times 5 \mu \mathrm{m}$. The root-mean-square (RMS) roughness value was calculated from the obtained image. The height and length of the regular microscale patterns was measured using a 3D microscope (Leica DVM 5000, Germany). FTIR spectra were recorded to determine the type of organic groups on the surfaces using the KBr pellet method (FT-IR; Nicolet Nexus670, USA). The chemical composition of the surfaces was analyzed via X-ray photoelectron spectroscopy (XPS) using an X-ray photoelectron spectrometer (XPS; AXIS UltraDLD, Kratos, Japan).

\section{Normal and low temperature wettability test}

The apparent contact angles and sliding angles on these surfaces of $4 \mu \mathrm{l}$ water droplets were directly measured using a contact angle analyser (Kruss DSA100, Germany). In order to ensure the accuracy of the apparent contact angle, each contact angle measurement was repeated at least three times, and then the average value was calculated.

To further examine low-temperature wettability, we conducted another experiment to test the superhydrophobic potential of each sample at low temperatures. The samples and deionized water were placed inside a refrigerator at $-18{ }^{\circ} \mathrm{C}$ for some time until the deionized water became supercooled. Then, we tried to simulate the conditions of freezing rain by spraying supercooled water droplets on the surfaces of the different samples. Using a camera with high magnification, we could quickly compare the different wettability properties of these samples.

\section{Ice adhesion strength test}

For performing ice adhesion strength measurements, we prepared a cuvette filled with distilled water ahead of time and gently placed a sample on the rabbet of the cuvette. This was kept in a refrigerator at $-18{ }^{\circ} \mathrm{C}$ for $24 \mathrm{~h}$ to form a steady ice column sticking to the sample surface. Subsequently, ice adhesion strength was measured with a previous self-made ice adhesion strength measurement device, which included a cooling plate with a wide range of temperatures. A force transducer was convenient to immediately record when the force successfully removed the ice column from the sample surfaces at the same position every time. Finally, ice adhesion strength of each sample was easily calculated by the formula of the stress and pressure. For the final values, experiments were repeated several times.

\section{Results and discussion}

\section{Surface morphology}

In this section, we constructed three superhydrophobic structured surfaces via three different treatments as mentioned above, with the objective to analyse and study the correlation between surface morphology, wettability, and anti-icing properties. Additionally, a smooth hydrophobic Al surface was fabricated as a standard control to compare with the three superhydrophobic surfaces in terms of anti-icing properties. The FESEM images of the three structured morphologies are shown in Fig. 2. It can be seen from the FESEM images (Fig. 2(a)) that the average size of the microscale array pattern is about $150 \mu \mathrm{m} \times 150 \mu \mathrm{m}$, with a spacing of 60-90 $\mu \mathrm{m}$. Fig. 2(b) shows the SEM images of sample 3, fabricated by alkali etching with subsequent boiling treatment. A large number of flake-like nanoparticles with a length of $\sim 100 \mathrm{~nm}$ were evenly distributed on the surface, and some sub-nanoscale flakes had aggregated
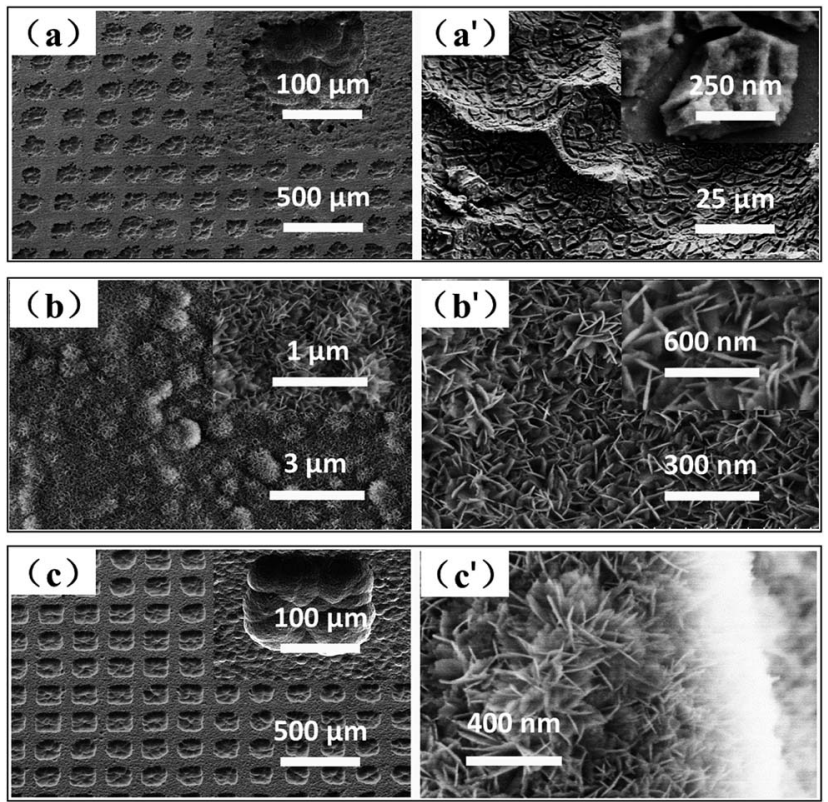

Fig. 2 SEM images of the hierarchical morphologies without modification obtained via three different treatments: (a) micropit-structured surface (sample 2); (b) nanoflake-structured surface (sample 3); (c) micro/nano-hierarchically-structured surface (sample 4). 
to form a series of three-dimensional spherical particles with a diameter of $1 \mu \mathrm{m}$, dotted on the pre-existing flake-like nanoparticle array. The micro/nano-hierarchically-structured surface was successfully obtained by the combination of two methods (lithography processing, alkali etching with subsequent boiling treatment). The microscale array pattern was completely covered by a layer of nanoflakes produced via alkali etching with subsequent boiling treatment, regardless of the position or the spacing of the micropits (see Fig. 2(c)).

In order to confirm the accurate size of the micropit, a 3D microscope was used. With the help of a professional analysis software, the depth and width of each micropit were determined to be approximately $37.468 \mu \mathrm{m}$ and $142.515 \mu \mathrm{m}$, respectively, as shown in Fig. 3(b).

To further investigate the superhydrophobic micro/nanohierarchically-structured surface, the 3D morphologies and the roughness of the superhydrophobic surface were successfully obtained from the AFM images shown in Fig. 4(a) and (b). It can be seen that a roughness of $17 \mathrm{~nm}$ is mainly caused by the large number of flake-like particles covering the structure. The spacing between the textural peaks of the roughness is vital to trap more air in the pockets formed by the micro/nano-structure. ${ }^{14}$ The characterization of the superhydrophobic surface provided a fundamental confirmation that the water droplets cannot penetrate into the pitch profile, resulting in a high static contact angle and low ice adhesion strength. ${ }^{15}$

\section{Chemical composition analysis}

An EDS spectrum (Fig. 5) was recorded to characterize the surface of the nanoflake-structured surface without modification (sample 3). Two clear peaks, ascribed to $\mathrm{Al}$ and $\mathrm{O}$, could be observed in the spectrum (Fig. 5(a)), indicating that sample 3 was mainly composed of $\mathrm{Al}$ and $\mathrm{O}$ (Fig. 5(b)). Furthermore, EDS mapping images, shown in Fig. 5(c), demonstrated that a large amount of $\mathrm{O}$ and $\mathrm{Al}$ was evenly distributed on the surface. Based on the features of the alkali treatment, the aluminum substrate

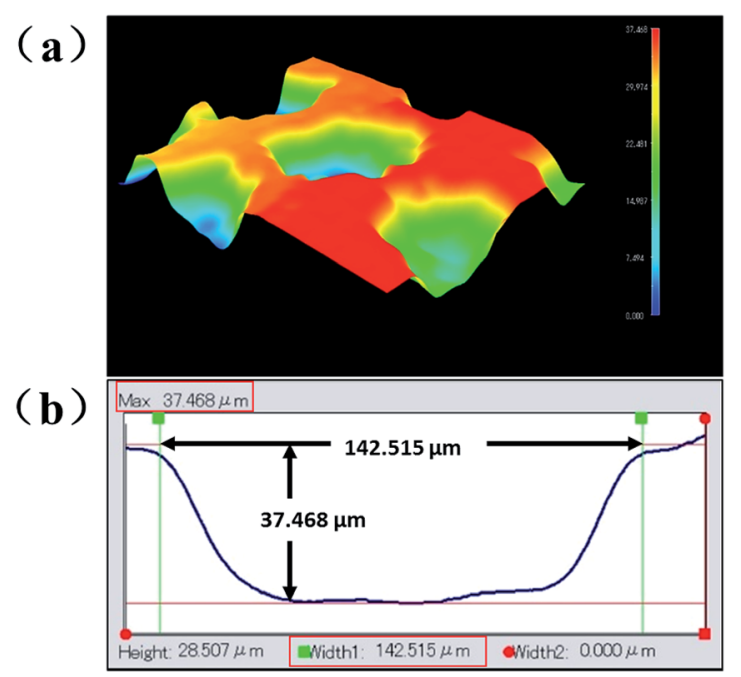

Fig. 3 (a) 3D surface topography and (b) width and depth of a micropit.

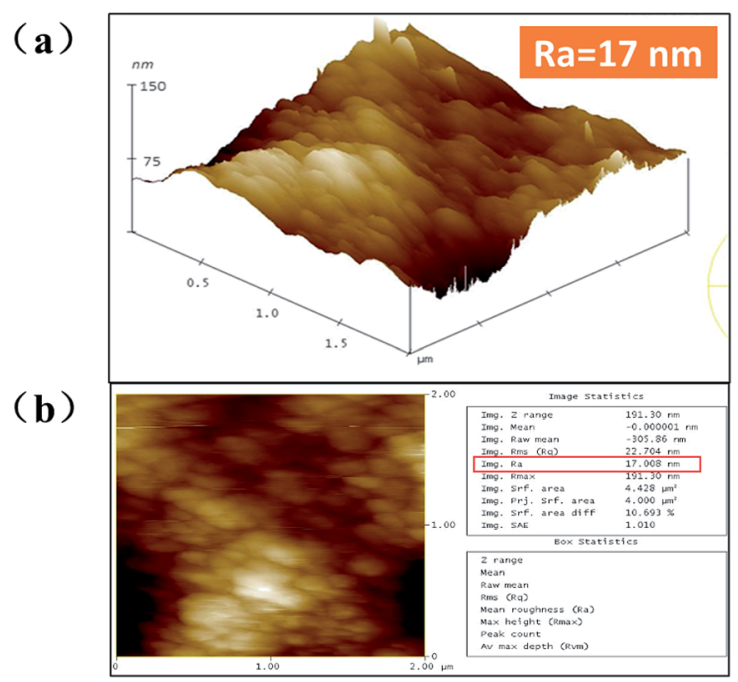

Fig. 4 AFM images of (a) surface height and (b) surface roughness of the nanoflake-structured Al surface without modification.
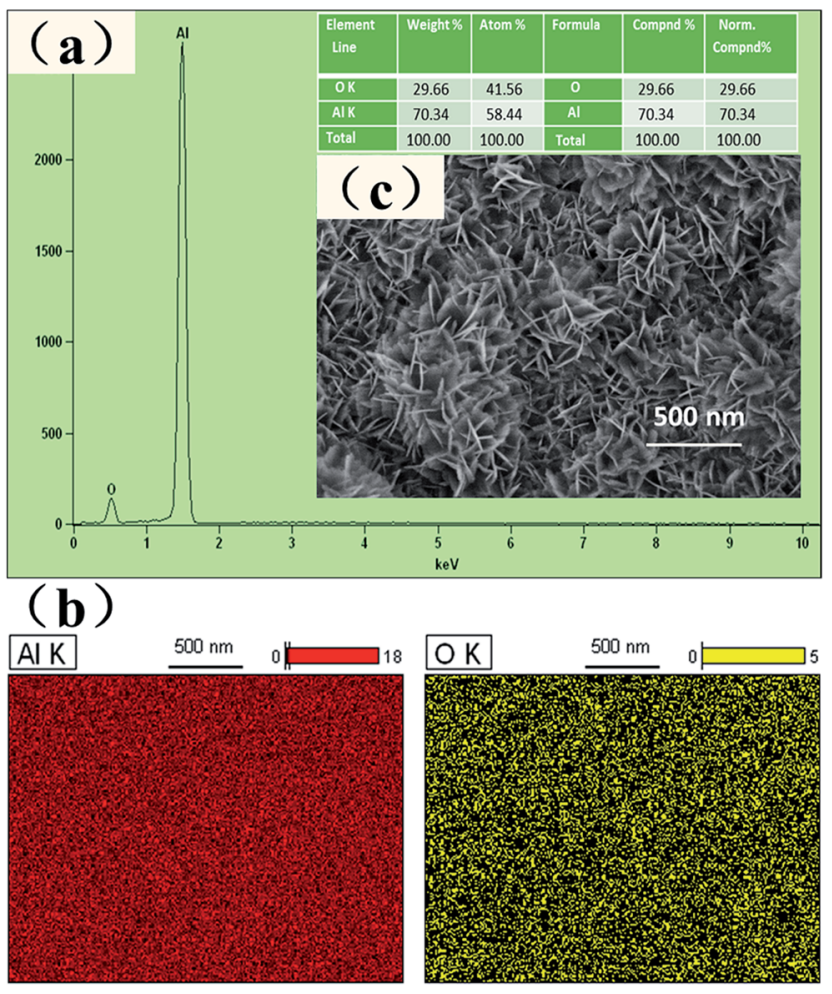

Fig. 5 (a) EDS spectrum; (b) elemental mapping of Al and $\mathrm{O}$ on the nanoflake-structured Al surface without modification; and (c) corresponding SEM image.

reacting with $\mathrm{NaOH}$ solution causes the rapid precipitation of $\mathrm{Al}(\mathrm{OH})_{3}$ at extreme $\mathrm{pHs} .{ }^{16} \mathrm{The} \mathrm{Al}(\mathrm{OH})_{3}$ solid precipitates readily absorb water molecules and form a gel. During the boiling water treatment, the aluminate ions crystallize to afford $\mathrm{Al}(\mathrm{OH})_{3} \cdot{ }^{17}$ The reacting processes can be expressed as follows:

$$
2 \mathrm{Al}+2 \mathrm{NaOH}+6 \mathrm{H}_{2} \mathrm{O} \rightarrow 2 \mathrm{Al}(\mathrm{OH})_{4}{ }^{-}+2 \mathrm{Na}^{+}+3 \mathrm{H}_{2}
$$




$$
\mathrm{Al}(\mathrm{OH})_{4}{ }^{-} \rightarrow \mathrm{Al}(\mathrm{OH})_{3}+\mathrm{OH}^{-}
$$

In order to obtain superhydrophobicity, modifying with FAS17 was necessary to gain a low-surface energy chemical component. The chemical component on the $\mathrm{Al}$ surface before and after the modification was identified via FT-IR spectra, as shown in Fig. 6. The absorption peaks at around 579.89 and $483.73 \mathrm{~cm}^{-1}$ are assigned to the $\mathrm{Al}-\mathrm{O}$ group of the nanoflakestructured Al surface, regardless of the modification, which again confirms the presence of nanoflakes composed of $\mathrm{Al}(\mathrm{OH})_{3}$. Moreover, the $\mathrm{O}-\mathrm{H}$ bond signal appeared at around $3425.77 \mathrm{~cm}^{-1}$, which are attributed to water absorption by $\mathrm{KBr}$ during the FT-IR spectra test. Upon comparing the two FT-IR patterns, shown in Fig. 6(a), it was evident that the spectrum of the superhydrophobic Al surface after modification displays different absorption peaks. The bands at 2923.78 and 2853.31 $\mathrm{cm}^{-1}$ correspond to the $\mathrm{C}-\mathrm{H}$ asymmetric and symmetric stretching vibrations, respectively. The band at $1630.74 \mathrm{~cm}^{-1}$ is ascribed to the $\mathrm{C}-\mathrm{H}$ stretching vibrations. The other two bands at 1401.82 and $748.42 \mathrm{~cm}^{-1}$ are assigned to the $\mathrm{C}-\mathrm{H}$ in-plane and out-plane flexural vibrations, respectively. The characteristic absorptions at $1062.91 \mathrm{~cm}^{-1}$ of C-F (1300-1050 $\left.\mathrm{cm}^{-1}\right)$ belonging to the $\mathrm{C}-\mathrm{F}$ stretching vibration of the $-\mathrm{CF}_{2}-$ and $-\mathrm{CF}_{3}$ groups are clearly visible, indicating that the superhydrophobic Al surface was covered by the fluoroalkylsilane (FAS-17) film successfully, which endows the nanoflake-structured Al surface with a very low surface energy. In the spectrum corresponding to the nanoflake-structured $\mathrm{Al}$ surface before modification, the absorption peak between 1300 and $1050 \mathrm{~cm}^{-1}$ were not observed, which shows the absence of the fluoroalkylsilane (FAS-17) film. These results indicate that a superhydrophobic surface in the Cassie state can be achieved by fabricating micro/ nano-hierarchically-structured surfaces modified with hydrophobic organic groups at ambient temperature. ${ }^{18}$

The chemical composition of the superhydrophobic surface modified with FAS-17 was further verified via XPS, as shown in

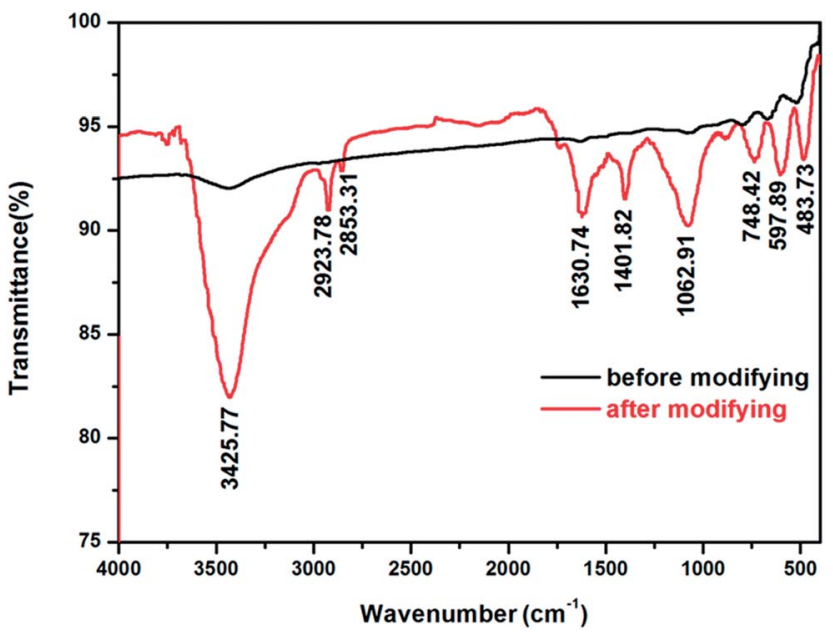

Fig. 6 FT-IR spectra of the superhydrophobic nanoflake-structured surface before and after modification.
Fig. 7. It can be seen that the sample possesses high-intensity peaks corresponding to F1s and FKLL and low-intensity peaks attributed to Si2p after modifying with FAS-17. Most importantly, the peaks corresponding to $-\mathrm{CF}_{2}$ (at $292 \mathrm{eV}$ ) were observed in the high resolution spectrum of C1s. Results indicated that the superhydrophobic surface was well-coated with fluoroalkylsilane (FAS-17).

\section{Normal and low temperature wettability contrast test}

The results for the apparent contact angle measurements present some evident differences before and after modification on sample 1 , sample 2 , sample 3 and sample 4 , as shown in Fig. 8. After comparing the apparent contact angle for the same sample before and after modification, we concluded that there is wider margin of improvement among these samples. The hydrophilic surfaces were immediately converted into

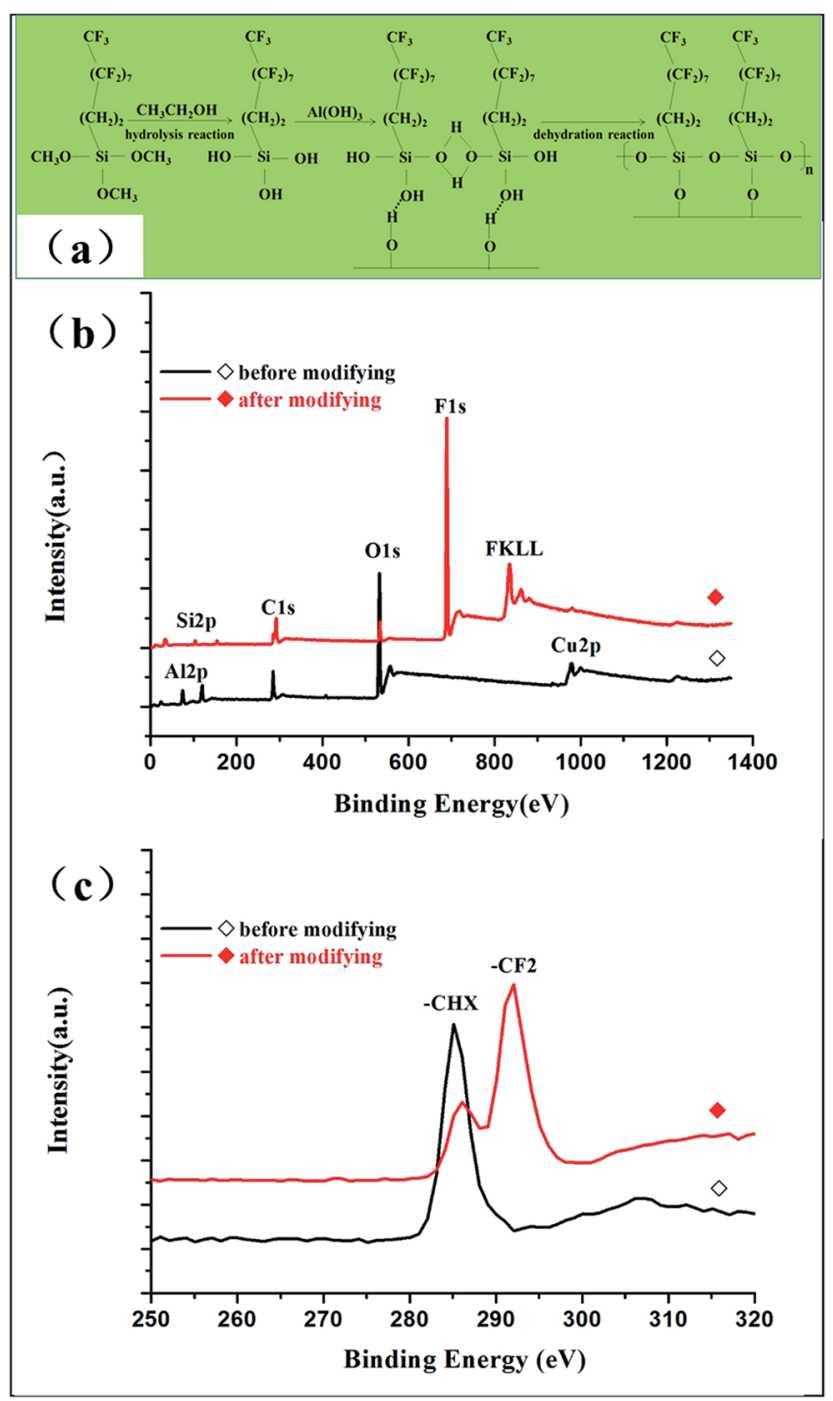

Fig. 7 (a) Chemical reaction process to construct a self-assembled superhydrophobic surface. (b) XPS spectra of the nanoflake-structured Al surface before and after modification. The actual composition measurement of the nanoflake structured Al surface; (c) magnification pattern of a partial region of the XPS spectra at high resolution. 
hydrophobic or superhydrophobic surfaces, especially sample 1, sample 3, and sample 4. For example, on the smooth substrate surface (sample 1), the apparent contact angle of a water droplet can be increased to about $120^{\circ}$ by lowering the surface free energy, which further confirms that superhydrophobic surfaces can be well established by introducing regularly-aligned closely-packed $\mathrm{CF}_{3}$ groups, with the lowest surface free energy, of $6.7 \mathrm{~mJ} \mathrm{~m}^{-2} .^{19}$ Moreover, sample 2 maintains the apparent contact angle at $130^{\circ}$, whether it was modified or not. This result shows that the dimensions or roughness of the micropit-structured surface is not suitable for the water droplets, as it causes the water droplets to completely impregnate the micropit surface to evolve into the Wenzel model. In addition, we can find that the apparent contact angle of superhydrophobic nanostructured surfaces (sample 3) reaches $169^{\circ}$, and the sliding angle is reduced to $6^{\circ}$. Moreover, we surprisingly discovered that the superhydrophobic hierarchically-structured surface (sample 4) exhibits higher non-wettability with apparent contact angles as high as approximately $173^{\circ}$ and sliding angles as low as about $4.5^{\circ}$ after modification. Compared with the other single-structured surfaces, the superhydrophobic properties of the nanoflakemicropit hierarchically-structured surface were better than those of any single-structured surface after modification. This result indicates that sample 3 and sample 4 have good superhydrophobic properties, such that the water droplet can easily roll off from these surfaces, which can be explained in detail by the Cassie-Baxter model, demonstrating that the microstructure of surfaces plays a key role in enhancing superhydrophobicity. ${ }^{20}$ The combination of micro-scale and nanoscale structures contributes significantly to entrap much more air, leading to the existence of a sealed air layer made of countless air pockets under the droplet. ${ }^{21}$

Considering the actual service conditions for aircrafts, the superhydrophobic properties at low temperatures were worth investigating by spraying supercooled water droplets on the four

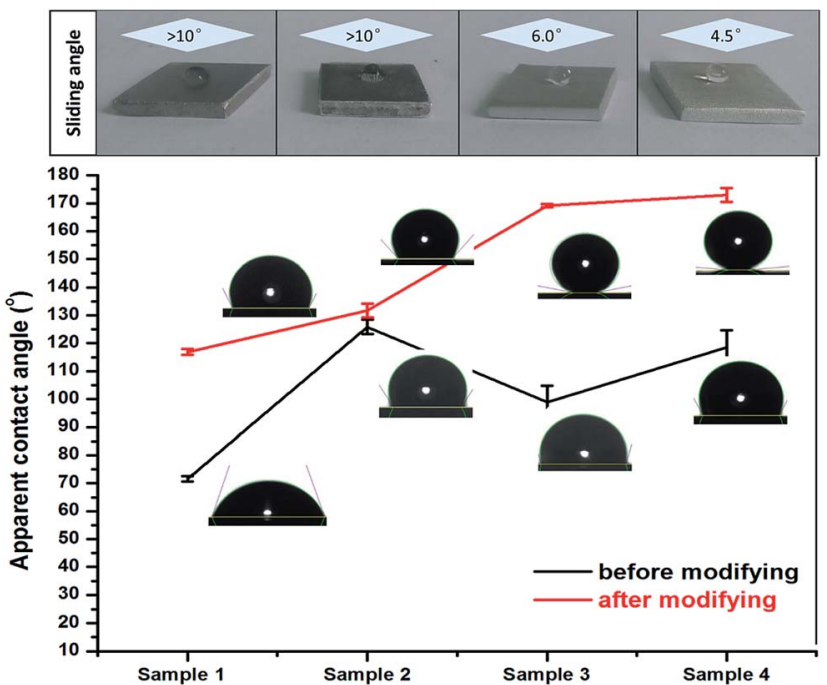

Fig. 8 Apparent contact angles and sliding angles of water droplets on the four surfaces before and after modification. different samples. Four samples with different structures were placed on a glass plate covered with a white filter paper at $-18{ }^{\circ} \mathrm{C}$ in the fridge. Fig. 9 depicts the results for the superhydrophobic properties, evaluated on sample 1, sample 2, sample 3, and sample 4 by spraying supercooled water droplets at a low temperature. We found that sample 3 and sample 4 can prevent the accumulation of supercooled water droplets, exhibiting a favourable superhydrophobic property. On the contrary, the supercooled water droplets sprayed on sample 1 and sample 2 revealed a hydrophilic characteristic to a certain extent. Clearly, the results convincingly demonstrate that the superhydrophobic surface with the micro/nano-structure effectively repelled the freezing water after being sprayed. Thus, the superhydrophobic surface with the micro/nanostructure may have more potential as an anti-icing material for aircrafts in the aviation industry.

\section{Ice adhesion strength}

To systematically evaluate the anti-icing properties, we tested the ice adhesion strength of the four surfaces at $-18^{\circ} \mathrm{C}$ by a selfmade measurement device, as shown in Fig. 10. The ice adhesion strength on the smooth surface (sample 1) was the highest, $710.5 \pm 38.9 \mathrm{kPa}$. For sample 2 , sample 3 and sample 4 , the ice adhesion strengths were $490.3 \pm 33.6 \mathrm{kPa}, 105.5 \pm 9.2 \mathrm{kPa}$ and $75 \pm 32.1 \mathrm{kPa}$, respectively. Obviously, the ice adhesion strength on the other surfaces is much lower than that on the smooth surface (sample 1), which confirms that both micro- and nanostructured surfaces with a rough texture retain more air pockets, minimizing the water-surface contact area. ${ }^{22}$ Moreover, we found that the ice adhesion strength on the micro-structured surface (sample 2) is significantly greater than that on both the nano-structured surface (sample 3) and the micro/nanostructured surface (sample 4). The ice adhesion on sample 2 is best described by the Wenzel model, whereby water infiltrates the spaces between surface features for a fully wetted surface. ${ }^{23}$ With the increase of the freezing time at lower temperatures, the greater ice adhesion strength could be due to the mechanical interlocking of ice in the micro-structured surfaces. ${ }^{24}$

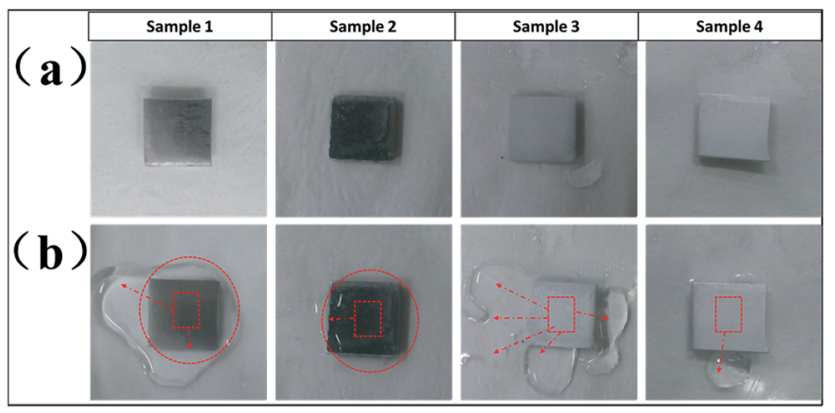

Fig. 9 Photographs of the superhydrophobicity test, conducted by spraying supercooled water droplets on the four surfaces at a low temperature: (a) before spraying supercooled water droplets; and (b) after spraying supercooled water droplets. (The red dotted box represents the contact point with the freezing rain movement and the red dotted circumference around the box represents the freezing rain movement range.) 


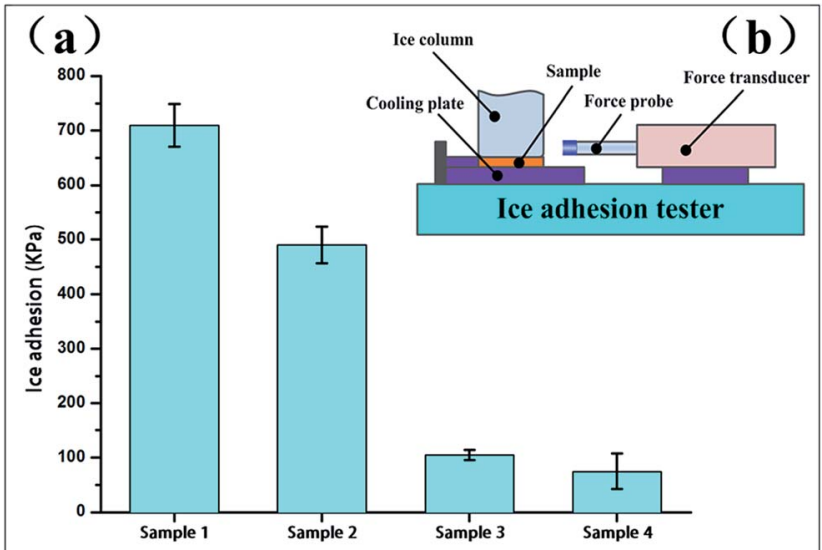

Fig. 10 (a) Ice adhesion strength on the four surfaces post-modification at $-18{ }^{\circ} \mathrm{C}$. (b) Schematic of ice adhesion strength measurement device.

Furthermore, the ice adhesion strength on the micro/nanostructured surface (sample 4) is only a little bit lower than on the nano-structured surface (sample 3 ). The variation between sample 3 and sample 4 could be explained by the actual contact interface between the ice/air or ice/hydrophobic surface interfaces. Although sample 3 and sample 4 both exhibit a CassieBaxter behavior, sample 4, with microscale array patterns and nanoflake structures, has the ability to induce more air pockets and even form an air layer to suspend the water droplets. Moreover, the layer of air can be maintained after freezing, and serves as a stress concentration position during the period of shear breakup. Thus, the air content between the solid surface and the ice directly determines ice adhesion strength and the capacity of resisting freezing rain. Based on the mechanism, sample 4 become relatively smaller, which causes to move away the ice column more easily. ${ }^{25}$

\section{Conclusion}

In summary, we tactfully designed and fabricated a superhydrophobic hierarchically-structured surface with anti-icing properties by the combination of lithography processing and chemical etching methods on the surface of an $\mathrm{Al}$ substrate. By the combination of these some simple processes, we successfully constructed a superhydrophobic hierarchically structured surface containing a micropit array pattern and nanoflakes with low surface energy. The superhydrophobic surface exhibited a stronger non-wettability than the other single-structured surfaces, with an apparent contact angle of up to $173^{\circ}$ and a sliding angle below $4.5^{\circ}$. Moreover, the superhydrophobic hierarchically structured surface exhibited a lower ice adhesion strength and prevented the supercooled water droplets from accumulating. All these results demonstrate that the superhydrophobic hierarchically-structured surface exerts a good water repellency and excellent anti-icing properties.

Although great progress has been made, few singlestructured surfaces have shown the ability to rapidly repel impacting and condensing water droplets, suppress ice nucleation and reduce ice adhesion, all while operating in various environments, and exhibiting high durability and longevity. Therefore, future research should focus on durability and longevity, rather than achieving maximum performance under ideal conditions. On the one hand, by incorporating hydrophobic lubricating layers, we can develop and design environmentally-friendly and mechanically-durable lubricantinfused superhydrophobic hierarchically structured surfaces with improved durability and longevity. On the other hand, we need to make comprehensive assessments of lubricant-infused superhydrophobic hierarchically-structured surfaces with respect to low temperature resistance, humidity tolerance, and durability during ice removal persists, when ice accretion occurs due to freezing rain, snow, in-cloud icing or fog icing, and frost formation. Furthermore, the integration of lubricantinfused superhydrophobic hierarchically structured surfaces with current aircraft anti-icing technologies has not been extensively studied, but it is an important consideration for eventual practical applications.

\section{Acknowledgements}

In this study, we sincerely express our greatest gratitude to some respected persons and research groups who gave us valuable guidelines and support, including Tutor professor Taojie, Senior Dr Shen and all colleagues of Taojie's group. This work was partially supported by the financial support of the National Science Foundation of China (No. 51671105), the National Postdoctoral Program for Innovative Talent (BX201600073), the NUAA Innovation Program for Graduate Education (kfj20160607), and the financial support of NUAA Innovation Practice and Free Exploration Program for College Education (ZT2015051).

\section{Notes and references}

1 M. J. Kreder, J. Alvarenga and P. Kim, Nat. Rev. Mater., 2016, 1, 15003.

2 J. Lv, Y. Song and L. Jiang, ACS Nano, 2014, 8, 3152-3169.

3 T. V. J. Charpentier, A. Neville and P. Millner, J. Colloid Interface Sci., 2013, 394, 539-544.

4 R. W. Gent, N. P. Dart and J. T. Cansdale, Philos. Trans. $R$. Soc., A, 2000, 358, 2873-2911.

5 L. B. Boinovich, A. M. Emelyanenko and V. K. Ivanov, ACS Appl. Mater. Interfaces, 2013, 5, 2549-2554.

6 K. Rykaczewski, S. Anand and S. B. Subramanyam, Langmuir, 2013, 29, 5230-5238.

7 S. Wang, K. Liu and X. Yao, Chem. Rev., 2015, 115, 8230-8293.

8 C. Hao, J. Li and Y. Liu, Nat. Commun., 2015, 6, 7986.

9 R. M. Fillion, A. R. Riahi and A. Edrisy, Renewable Sustainable Energy Rev., 2014, 32, 797-809.

10 K. Rykaczewski, S. Anand and S. B Subramanyam, Langmuir, 2013, 29, 5230-5238.

11 Y. Wu, W. Zhao and W. Wang, RSC Adv. , 2016, 6, 5100-5110.

12 T. Cheng, R. He and Q. Zhang, J. Mater. Chem. A, 2015, 3, 21637-21646. 
13 L. B. Boinovich, A. M. Emelyanenko and K. A. Emelyanenko, Phys. Chem. Chem. Phys., 2016, 18, 3131-3136.

14 B. Bhushan, Y. C. Jung and K. Koch, Philos. Trans. R. Soc., A, 2009, 367, 1631-1672.

15 L. Yin, L. Zhu and Q. Wang, ACS Appl. Mater. Interfaces, 2011, 3, 1254-1260.

16 J. Zhang, M. Klasky and B. C. Letellier, J. Nucl. Mater., 2009, 384, 175-189.

17 J. Li, C. A. Prestidge and J. Addai-Mensah, J. Colloid Interface Sci., 2000, 224, 317-324.

18 Y. Xiu, L. Zhu and D. W. Hess, Nano Lett., 2007, 7, 3388-3393.

19 M. Miwa, A. Nakajima and A. Fujishima, Langmuir, 2000, 16, 5754-5760.
20 S. Guo, L. Wang and E. Wang, Chem. Commun., 2007, 30, 3163-3165.

21 S. Wang, K. Liu and X. Yao, Chem. Rev., 2015, 115, 82308293.

22 A. Cassie and S. Baxter, Trans. Faraday Soc., 1944, 40, 546551.

23 R. N. Wenzel, Ind. Eng. Chem., 1936, 28, 988-994.

24 D. K. Sarkar and M. Farzaneh, J. Adhes. Sci. Technol., 2009, 23, 1215-1237.

25 A. J. Meuler, G. H. McKinley and R. E. Cohen, ACS Nano, 2010, 4, 7048-7052. 